Wiser Healthcare and School of Public Health, University of Sydney, Australia

2 Department of Anaesthesia and Intensive Care, Western Health, Melbourne, Australia; Department of Critical Care, University of Melbourne Australia: School of Public Health, University of Sydney, Australia

alexandra.barratt@sydney.edu.au Cite this as: BMJ 2021;375:n2407 http://dx.doi.org/10.1136/bmj.n2407 Published: 04 October 2021

\section{Overdiagnosis is increasing the carbon footprint of healthcare}

\author{
Tackling overdiagnosis takes on a new urgency in the context of a climate emergency \\ Alexandra Barratt, ${ }^{1}$ Forbes McGain²
}

Clinical practice varies-some is excellent, delivering high quality care, improving health outcomes, and representing excellent value for money. But research over the past two decades has established that a significant minority of clinical care is low value, wasteful, or harmful. ${ }^{1}$

At the extreme end of low value care is no value care-otherwise known as overdiagnosis.

Overdiagnosis is the detection of harmless conditions that could be safely left undiagnosed and untreated. ${ }^{23}$ Once diagnosed, however, a cascade of tests and treatment usually follows, which will not benefit patients who were not unwell to start with. While we acknowledge that identifying individual cases of overdiagnosis is challenging (or impossible), what is clear is that it's real and widespread, turning many people into patients unnecessarily. Globally, 500 ooo people a year are overdiagnosed with thyroid cancer, including in low and middle income countries $^{45}$; 70 ooo women a year may be overdiagnosed with breast cancer in the US, and 4000 a year in Australia; and 8000 Australian men a year are overdiagnosed with prostate cancer. ${ }^{67}$

Overdiagnosis affects all ages and life stages. ${ }^{8-13}$ In the UK, the problem of overdiagnosis and overtreatment is of such concern to the Royal College of General Practitioners that a standing group on overdiagnosis has been established, which has advanced recommendations to try to combat it. ${ }^{14}$ While a growing list of overdiagnosed conditions is recognised, the burden that patients' overdiagnoses places on healthcare services and staff is easily overlooked.

Recently Tennison and colleagues examined carbon dioxide equivalent ( $\mathrm{CO}_{2} \mathrm{e}$ ) emissions in the NHS over three decades, 1990 to 2019, finding that the service had reduced CO2e emissions by $26 \%$-a world leading achievement. ${ }^{15}$ Episodes of inpatient care doubled over the same period and health spending tripled in real terms, even though the population only increased by $17 \%$. Some of the additional episodes of care reflect changing population demographics and increased appropriate diagnoses, but some undoubtedly were caused by overdiagnosis. By definition, overdiagnosis generates carbon emissions without improving health.

Novel efforts to curb overdiagnosis are underway, yet so far have had limited uptake. ${ }^{16-18}$ Tackling it, however, takes on a new urgency in the context of the climate emergency. Carbon emissions for zero gain in health outcomes become intolerable.

The rise and rise of overdiagnosis-and its corollaries, over-testing and overtreatment-has unfolded synchronously with the health emergency caused by climate change. Climate change will drive ever more people to seek medical care for exposure to extreme heat, drought, floods, fire and extreme weather, vector borne diseases, air pollution, and disruptions to food and electricity supply. ${ }^{19}$ The additional burden of more patients because of overdiagnosis will be in addition to these surges of climate change driven demand for healthcare. The combined effect of both threatens to overwhelm already overstretched health systems across the world.

The NHS has set ambitious targets to reach zero carbon healthcare by 2040 . The NHS England data reported by Tennison and colleagues are revealing: they show that most of the reduction in $\mathrm{CO}_{2} \mathrm{e}$ emissions between 1990 and 2019 has come from decarbonisation of UK energy (with large reductions in building energy emissions) and from changing the propellants in respiratory metered dose inhalers. CO2e emissions generated from provision of clinical care and its associated activities have generally remained stable or increased. Emissions from medical instruments have remained stable and emissions from commissioned care (clinical services procured by the NHS from private healthcare providers) increased fivefold. Between 2001 and 2012 total NHS England emissions increased slightly as investment in healthcare expanded from $4.7 \%$ to $7.6 \%$ of gross domestic product, before falling again in line with ongoing decarbonisation of UK electricity. ${ }^{15}$

To make further and sustained cuts to healthcare's carbon footprint will therefore require changes to models of clinical care. Tackling overdiagnosis and over-treatment will be a key step.

Reducing overdiagnosis and over-treatment is an untapped reservoir of potential to cut healthcare's carbon emissions, while at the same time safeguarding the quality of care and ensuring that patient outcomes aren't adversely affected. A valuable co-benefit will be reductions in healthcare costs.

Clinicians and patients care deeply about climate change and many clinicians are already implementing change. The Gassing Greener project encourages anaesthesiologists to use less desflurane and nitrous oxide, and track their carbon performance. ${ }^{20}$ Re-usable rather than single use anaesthetic and surgical equipment, such as gowns, can reduce emissions in places where there is decarbonised electricity supply, at equivalent or lower cost. ${ }^{21-23}$ There are many more opportunities for clinicians to green clinical care. ${ }^{24-26}$

The desire to green clinical care could motivate and sustain clinicians in tackling the challenges of overdiagnosis. Knowing that reducing overdiagnosis has the potential to reduce emissions and conserve resources (financial and human) while safeguarding 
the quality and safety of healthcare may make dealing with it more salient and rewarding.

Concerted policy action and clinical leadership is needed to enable and accelerate initiatives to deal with overdiagnosis and healthcare's carbon emissions. In a climate emergency, it's vital this happens. The seeds of change are there.

Competing interests: $\mathrm{AB}$ receives grants from the National Health and Medical Research Council of Australia and was a member of the Scientific Committee of Preventing Overdiagnosis international conferences 2013-2021. FMcG has received grants from the Australian Medical Research Future Fund and the Australian and New Zealand College of Anaesthetists. He receives royalties for co-inventing a device that reduces aerosol exposure (the McMonty hood).

Commissioned, not peer reviewed.

1 Braithwaite J, Glasziou P, Westbrook J. The three numbers you need to know about healthcare: the 60-30-10 Challenge. BMC Med 2020;18:102.

doi: 10.1186/s12916-020-01563-4 pmid: 32362273

2 Carter SM, Rogers W, Heath I, Degeling C, Doust J, Barratt A. The challenge of overdiagnosis begins with its definition. BMJ2015;350:h869. doi: 10.1136/bmj.h869 pmid: 25740625

3 Brodersen J, Schwartz LM, Heneghan C, O'Sullivan JW, Aronson JK, Woloshin S. Overdiagnosis: what it is and what it isn't. BMJ Evid Based Med 2018;23:1-3. doi: 10.1136/ebmed-2017-110886 pmid: 29367314

4 Vaccarella S, Franceschi S, Bray F, Wild CP, Plummer M, Dal Maso L. Worldwide thyroid-cancer epidemic? the increasing impact of overdiagnosis. N Engl J Med 2016;375:614-7. doi: 10.1056/NEJMp1604412 pmid: 27532827

5 Lortet-Tieulent J, Franceschi S, Dal Maso L, Vaccarella S. Thyroid cancer "epidemic" also occurs in low- and middle-income countries. Int J Cancer 2019;144:2082-7. doi: 10.1002/ijc.31884 pmid: 30242835

6 Bleyer A, Welch HG. Effect of three decades of screening mammography on breast-cancer incidence. N Engl J Med 2012;367:1998-2005. doi: 10.1056/NEJMoa1206809 pmid: 23171096

7 Glasziou PP, Jones MA, Pathirana T, Barratt AL, Bell KJL. Estimating the magnitude of cancer overdiagnosis in Australia. Med J Aust2020;212:163-8. doi: 10.5694/mja2.50455 pmid: 31858624

8 Kazda L, Bell K, Thomas R, McGeechan K, Sims R, Barratt A. Overdiagnosis of attention-deficit/hyperactivity disorder in children and adolescents: a systematic scoping review. JAMA Netw Open2021;4:e215335. doi: 10.1001/jamanetworkopen.2021.5335 pmid: 33843998

9 Cundy T, Ackermann E, Ryan EA. Gestational diabetes: new criteria may triple the prevalence but effect on outcomes is unclear. BMJ2014;348:g1567. doi: 10.1136/bmj.g1567 pmid: 24618099

10 Copp T, Jansen J, Doust J, Mol BW, Dokras A, McCaffery K. Are expanding disease definitions unnecessarily labelling women with polycystic ovary syndrome?BM/2017;358:j3694. doi: 10.1136/bmj.j3694 pmid: 28814559

11 Bell K, Doust J, McGeechan K, etal. The potential for overdiagnosis and underdiagnosis because of blood pressure variability: a comparison of the 2017 ACC/AHA, 2018 ESC/ESH and 2019 NICE hypertension guidelines. J Hypertens 2021;39:236-42 doi: 10.1097/HJH.0000000000002614 pmid: 32773652

12 Bell KJL, Doust J, Glasziou P, etal. Recognizing the potential for overdiagnosis: Are high-sensitivity cardiac troponin assays an example?Ann Intern Med 2019;170:259-61. doi: 10.7326/M18-2645 pmid: 30716768

13 Brownlee S, Chalkidou K, Doust J, etal. Evidence for overuse of medical services around the world. Lancet 2017;390:156-68. doi: 10.1016/S0140-6736(16)32585-5 pmid: 28077234

14 Treadwell J, McCartney M. Overdiagnosis and overtreatment: generalists--it's time for a grassroots revolution. Br J Gen Pract 2016;66:116-7. doi: 10.3399/bjgp16X683881 pmid: 26917633

15 Tennison I, Roschnik S, Ashby B, etal. Health care's response to climate change: a carbon footprint assessment of the NHS in England. Lancet Planet Health 2021;5:e84-92. doi: 10.1016/S2542-5196(20)30271-0 pmid: 33581070

16 Ahn HS, Welch HG. South Korea's thyroid-cancer "epidemic"-turning the tide. N Engl J Med 2015;373:2389-90. doi: 10.1056/NEJMc1507622 pmid: 26650173

17 Doust J, Vandvik PO, Qaseem A, etalGuidelines International Network (G-I-N) Preventing Overdiagnosis Working Group. Guidance for Modifying the Definition of Diseases: A Checklist. JAMA Intern Med 2017;177:1020-5. doi: 10.1001/jamainternmed.2017.1302 pmid: 28505266

18 Nickel B, Moynihan R, Barratt A, Brito JP, McCaffery K. Renaming low risk conditions labelled as cancer. BM/2018;362:k3322. doi: 10.1136/bmj.k3322 pmid: 30100549

19 The Lancet. Climate and COVID-19: converging crises. Lancet 2021;397:71. doi: 10.1016/S0140-6736(20)32579-4 pmid: 33278352

20 Yale Center on Climate Change and Health. https://ysph.yale.edu/climate/phes/challenge.

21 McGain F, Story D, Lim T, McAlister S. Financial and environmental costs of reusable and single-use anaesthetic equipment. Br J Anaesth 2017;118:862-9. doi: 10.1093/bja/aex098 pmid: 28505289

22 Sherman JD, Raibley LA, 4thEckelman MJ. Life cycle assessment and costing methods for device procurement: comparing reusable and single-use disposable laryngoscopes. Anesth Analg 2018;127:434-43. doi: 10.1213/ANE.0000000000002683 pmid: 29324492
23 Overcash M. A comparison of reusable and disposable perioperative textiles: sustainability state-of-the-art 2012. Anesth Analg 2012;114:1055-66. doi: 10.1213/ANE.0b013e31824d9cc3 pmid: 22492184

24 McGain F, Muret J, Lawson C, Sherman JD. Environmental sustainability in anaesthesia and critical care. Br J Anaesth 2020;125:680-92. doi: 10.1016/j.bja.2020.06.055 pmid: 32798068

25 Sherman JD, Thiel C, MacNeill A, etal. The green print: advancement of environmental sustainability in healthcare. Resour Conserv Recycling 2020;161:104882doi: 10.1016/j.resconrec.2020.104882

26 Barraclough KA, Agar JWM. Green nephrology. Nat Rev Nephrol 2020;16:257-68. doi: 10.1038/s41581-019-0245-1 pmid: 32034297 